\title{
Organisationsformen der balinesischen Gesellschaft
}

\section{Einleitung}

Welche Lehrmeinungen Gelehrte auch immer vertreten mögen, stimmen sie wenigstens in einem überein, nämlich darin, daß die Formen der balinesischen Gesellschaftsordnung eine enorme Variationsbreite aufweisen. Innerhalb der Vielfalt von Erscheinungsformen einen gemeinsamen Nenner zu finden, läßt selbst anerkannte Balikenner fast verzweifeln, denn «Neither simplicity nor uniformity are Balinese virtues» (C. GEERTZ 1959: 991), «La complexité de la société balinaise est notoire» (GUERMONPREZ 1980:37) oder "The social organization [...] is, in fact, heterogeneous and confusing in respect to its essential aspects, and at times completely differing. We are dealing here with what I would call an cordered anarchy'» (SCHAAREMAN 1986: 141). Der verwirrenden Vielfalt sind sich auch die Balinesen bewußt. Sie drücken dies für die dörfliche Sphäre mit der Metapher: «anderes Dorf, anderes adat» ("len desa, len adat») aus. Doch nicht genug mit den Unterschieden von Dorf zu Dorf. Weitere Adatbereiche regeln beispielsweise das Handeln der Akteure innerhalb ihrer Familien bzw. Verwandtschaftsgruppen, in Bewässerungsgemeinschaften, Freiwilligenorganisationen und - auf höherer Maßstabsebene - in den Untertanengebieten früherer Königreiche. Einer der Forscher, der bestrebt ist, über bloße Beschreibung von Phänomenen der komplexen Sozialorganisation hinauszugehen und diese als Resultat einer prozeßhaften Entwicklung in einem Kontinuum zu deuten, ist HOWE (1989: 47-71). Teile des vorliegenden Artikels folgen denn auch seiner Argumentation.

Einleitend soll der zentrale Begriff adat verdeutlicht und dessen Veränderungen über den Raum und die Zeit dargelegt werden. Weil bei den zumeist in Bergdörfern lebenden bali aga bis heute zahlreiche sozio-kulturelle Merkmale der ursprünglichen balinesischen Gesellschaft erkannt werden können, soll vorerst dieser Gruppierung Beachtung geschenkt werden. Anschließend wird auf Fremdeinflüsse aus dem hindujavanischen Königreich Majapahit verwiesen, die dem balinesischen Substrat von Gemeinschaften in den Tieflandebenen vor allem ab Mitte des 14. Jahrhunderts aufgepfropft worden sind. Da sich die Ideologie hierarchischer Abstammungsgruppen im balinesischen Kastensystem ausdrückt, sollen dessen Gliederungsprinzipien sowie Regeln und Praktiken der Interaktion zwischen Angehörigen unterschiedlicher Kasten und Positionen aufgezeigt werden. Im folgenden wird auf balinesische Gruppierun- gen verwiesen, die nach der extern induzierten Rückstufung in die vierte Kaste darnach trachteten, ihre Position durch Bezugnahme auf den javanischen Hochadel anzuheben. Der scheinbar klar strukturierten und hierarchisierten Gesellschaft steht die Tatsache gegenüber, daß Balinesen ebenso abstammungs- und kastenübergreifenden Gruppierungen angehören: Sie waren Untertanen von um Macht rivalisierenden Herrschern; sie sind Mitglieder von Tempelkongregationen, Dorfteilorganisationen, Dorfgemeinschaften und können Bewässerungsgenossenschaften angehören, denen egalitäre Prinzipien zugrunde liegen. Damit wird nicht nur das Spannungsfeld der balinesischen Gesellschaftsordnung deutlich gemacht, sondern ebenso ein Charakteristikum der Inselkultur vor Augen geführt: das Streben nach Harmonisierung scheinbar unüberbrückbarer Gegensätze.

Das von den Vorfahren vermittelte und übernommene Wissen wird adat genannt. Das Wort ist arabischen Ursprungs und beinhaltet "Gewohnheit», «Sitte», «Brauch». Im islamischen Rechtssystem handelt es sich dabei um das Gewohnheitsrecht, das in jenen Fällen zur Anwendung gelangt, die mit Hilfe der autorisierten Gesetzesquellen nicht eindeutig gelöst werden können. Im indonesischen Sprachgebrauch ist der Sinngehalt von adat umfassender und wird von ALISJAHBANA (1966:3-5) wie folgt umschrieben: «Like other early traditional cultures in history, the Indonesian people, prior to the arrival of Indian culture, had evolved a style of thinking at once complex, all-inclusive, and highly intuitive. This style of thought was closely bound up with the enormously important position of religion in the cultural life of Indonesian society (...). Most of his (i. e. man's) knowledge and his arts were subsumed in the intellectual legacy he had received from his forefathers, and which he called adat. This adat was very different from what we call custom or convention today. Its meaning was not simply wider, but more particularly went far deeper. It included everything we call law nowadays; and it went much further than law in determining the needs and the actions of individuals and the community. It ordained the ceremonies of marriage, birth and deaths, the times and the methods for sowing rice, building a house, praying for rain, and many other things. (...) Indeed from one point of view, adat was

Albert Leemann, Prof., Dr., Geograph. Institut der Universität Zürich-Irchel, Winterthurerstr. 190, 8057 Zürich 
simply a social expression of the community religion, in as much as it was not a human creation, and in its exercise men were still constantly watched over by the spirits and supernatural powers ruling the community."

"Because the adat which regulated the entire life of the community was dominated by spirits and supernatural powers, that communal life was inevitably static and deeply conservative. Its roots lay in the obscurity of the past, when the ancestors laid down the adat once and for all, or as the Minangkabau say: It doesn't crack with the heat or rot in the rain.) In such an environment the word (old) had a special significance, denoting something venerable, sacred, powerful and full of wisdom.»

Wenn auch das adat idealtypisch ein für allemal fixierte Handlungs- und Verhaltensmaximen beinhaltet und unabdingbar mit dem Glauben verknüpft ist, ist es in Wirklichkeit nicht statisch, sondern über Zeit und Raum Änderungen unterworfen. Ursachen dafür können entweder gesellschaftsintern induzierte Innovationen oder aber externe Rahmenzwänge sein, welche Wandel stimulieren bzw. erzwingen. Nicht in allen Regionen Balis konnten javanische Statthalter der Majapahit-Könige ab Mitte 14. Jahrhundert ihren Einfluß gleichermaßen durchsetzen und sich die hindujavanisch geprägte Kasten- und Klassenstruktur etablieren. Nicht überall und nicht gleichzeitig sind dörfliche Wirtschaftseinheiten gleichermaßen in übergeordnete Wirtschaftssysteme einbezogen worden. Änderungen im Verwaltungsbereich während der niederländischen Kolonialherrschaft auf Bali (ab Mitte 19. Jahrhundert in Nordbali bzw. ab Beginn 20. Jahrhundert in Südbali) haben Eigenbefugnisse einheimischer Gemeinschaften mehr oder weniger stark beschnitten. Rezente Vereinheitlichungsmaßnahmen im Rahmen gesamtindonesischer Modernisierungsprogramme, verbunden mit zunehmenden Urbanisierungsprozessen, wirken sich in der Gegenwart dahingehend aus, daß gewisse Adatelemente durch den Staat in Frage gestellt und durch Einheitsnormen ersetzt werden. Friktionen unter Mitgliedern einer Gemeinschaft können sich dann ergeben, wenn das adat - nicht zuletzt aufgrund seiner integrierten religiösen Komponente - sich langsamer wandelt als die externen Rahmenbedingungen, wenn «Traditionalisten" an der Unverrückbarkeit des adat festhalten, «Modernisten» aber neue Freiräume zu ihren Gunsten zu nutzen trachten.

Auf Fahrten von der Südküste ins Inselinnere fallen jedem Balibesucher markante Unterschiede in der Kulturlandschaft auf. In der gut erschlossenen, dicht besiedelten Ebene säumen Kokospalmhaine die Küste, die von vorbildlich bewässerten Naßreiskomplexen abgelöst werden, wo sich Dorf an Dorf reiht und sich die höfischen Zentren befinden. Hier ist die Bevölkerung in Kasten gegliedert und hierarchisch klar strukturiert. Diese Zone wird oft «Wiege der hindubalinesischen Kultur» genannt. Oberhalb einer Höhe von rund $600 \mathrm{~m}$ - wie auch an küstennahen Steilhängen - wird der Bewässerungsfeldbau durch Trockenfeldbau abgelöst. In dieser Übergangszone von der Ebene zum Bergland sind Mais und Knollenfrüchte Hauptanbauprodukte. Der Zugang zu den auf
Spornen zwischen tiefen Schluchten liegenden Dörfern war - und ist teilweise noch heute - vor allem in der Regenzeit erschwert. Die Bevölkerungsdichte nimmt ab. Wenn auch diese Dörfer in Beziehung mit der Wirtschaft und den Märkten in der Ebene stehen, ist die meistens aus commoners zusammengesetzte Dorfbevölkerung gesellschaftlich und kulturell eher bergwärts ausgerichtet. Im anschließenden Bergland sind bereits in der ersten Hälfte des 19. Jahrhunderts, versteckt unter schattenspendenden Bäumen, Kaffeepflanzungen, später Obstund Gemüsegärten und neuerdings vor allem Gewürznelkenhaine angelegt worden. In Gebieten extensiver Bewirtschaftung werden balinesische Rinder und gelegentlich auch Ziegen gezüchtet. Die Gebirgsflanken schließlich sind teilweise noch immer mit tropischem Regenwald bestockt. Das Bergland ist das hauptsächliche Siedlungsgebiet der sogenannten «ursprünglichen Balinesen" (bali aga; syn. bali mula). Dieser Begriff ist insofern irreführend, als diese Gruppe zwar viele ursprüngliche Merkmale mit ostindonesischen Völkern gemeinsam hat, in einer Frühphase aber doch bhairavabuddhistischen und hinduistischen Einflüssen ausgesetzt war, welche das Kultleben auch ohne Kastenbildung beeinflußten. Die hindujavanische Prägung während und nach der Majapahitzeit hat die Dorfgemeinschaft der bali aga weniger stark betroffen. Hinweis dafür ist beispielsweise das Fehlen der Kremierung Verstorbener. Tote werden erdbestattet bzw. wurden - bis vor kurzem - wie in Sembiran in eine Schlucht geworfen oder wie in Trunyan auf Felsen ausgesetzt. Diese peripheren Dorfgesellschaften weisen in der Tat zahlreiche Merkmale ursprünglicher indonesischer Sozialorganisationen auf wie etwa die Rangierung innerhalb einer Gruppe aufgrund des Alters und das Bestreben nach Verwirklichung egalitärer Grundsätze innerhalb der Dorfgemeinschaft.

\section{Zur Gesellschaftsorganisation der Bergbevölkerung}

Ohne geodeterministischem Gedankengut zu verfallen, gilt es doch festzuhalten, daß die angesprochene naturund kulturräumliche Vielfalt Balis der Formung unterschiedlicher Wirtschafts- und Sozialorganisationen förderlich ist, denn die Arbeitstechniken und Organisationsformen bäuerlicher Gesellschaften haben nebst anthropogenen auch variablen natürlichen Rahmenbedingungen Rechnung zu tragen. Wenn wir uns nun kurz den spärlich besiedelten Berggebieten zuwenden, fällt deren periphere Lage zu den politischen Macht- und Wirtschaftszentren in der Ebene auf. Kommunikation, Austausch von Waren und Dienstleistungen zwischen der Peripherie und dem Palast des Herrschers als Zentrum waren - wenn auch nicht verunmöglicht - doch erschwert, was einer straffen Einbindung der Bergdörfer in die sozio-ökonomischen und sozio-politischen Netzwerke tiefer gelegener Gebiete hinderlich war. Aber auch das vom Tiefland stark abweichende Wirtschaftssystem und die schwächere ökonomische Basis trugen das ihre zum 
geringeren Bestreben der Krone nach einer totalen Unterwerfung und straffen Kontrolle der Berggebiete bei. Die Herrscher konzentrierten sich vielmehr auf die Absicherung gewinnversprechenderer Landstriche in der Ebene und in der Bergfußregion. Dieses Bestreben nach Beherrschung der von rivalisierenden Machthabern begehrten Gunstzonen absorbierte weitgehend die aus Untertanen rekrutierten Streitkräfte, die somit für aufwendige Feldzüge in das wirtschaftlich weniger attraktive Gebirge fehlten. Daß die bali aga aber trotzdem nicht in geschlossenen Dörfern, von den tiefer liegenden Zentren völlig abgekoppelt, lebten, geht aus neuen Untersuchungen hervor. So kann der Nachweis erbracht werden, daß Dorfgemeinschaften an der Südflanke des Vulkans Batur gelegentlich zu Frondiensten am Fürstenhof von Bangli verpflichtet wurden und Grenzschutzfunktionen für den Herrscher wahrzunehmen hatten. In bescheidenem Aus$\mathrm{maß}$ existierten ab dem 19. Jahrhundert auch gewisse ökonomische Einbindungen der Berggebiete in den überregionalen Handel, weiß man doch, daß mit königlicher Bewilligung eingesetzte Hafenmeister (ind.: syahbandar; bal.: subandar) nebst Tieflandprodukten und Sklaven ebenso Vieh aus dem Hochland vermarkteten. So konnten sich die im Süden lebenden Herrscher ohne volle territoriale Beherrschung und ohne direkte Steuererhebungen gewissen Nutzen und bescheidene Profite aus den Berggebieten sichern. Nur zu leicht übersieht man die Tatsache, daß sich in früheren Zeiten die Machtposition der Herrscher weniger durch die Fläche ihres Einflußbereiches als vielmehr durch die Anzahl ihrer Untertanen manifestierte. Der Einbezug von Bergbewohnern in rituelle Verpflichtungen am Hofe und militärische Dienste war auch deshalb weniger intensiv als in der Ebene, weil hier keine adeligen Statthalter (punggawa) der Könige wohnten, welche die bali aga kontrollierten. Ebensowenig zogen diese stärker nach innen gerichteten Dorfgemeinschaften Brahmanenpriester (padanda) für ihre religiösen Rituale bei. Supradörfliche Verbünde fehlen weitgehend. Um so auffallender ist es, daß voneinander getrennte Siedlungen viele kulturelle Gemeinsamkeiten aufweisen. Diese Tatsachen mögen Hinweis darauf sein, daß gewisse Grundmuster der $\mathrm{Aga}$ gemeinschaften für ganz Bali (und weitere östliche Inseln) bestimmend waren. ${ }^{\prime}$ Daß sich bali aga-Dörfer insgesamt von Dörfern in der Ebene abheben, darf keinesfalls in den unterschiedlichen physisch-geographischen Rahmenbedingungen allein gesucht werden, sondern muß ebenso die geringeren wandelinduzierenden Einflüsse aus den Feudalsystemen der Ebene einbeziehen. Dieser schwächere Durchdringungsgrad erleichert das Überleben althergebrachter Institutionen. Da die Kastenhierarchie in Agadörfern fehlt, entfallen hier auch Titelgruppen, die an den Adel gebunden sind, und das damit verbundene Statussymbol der balinesischen Hochsprache (alus) (HOWE 1989: 56 f.). Wenn im Idealfall des bali aga-Modells Ursprungsort, Abstammungsgruppe und Dorfterritorium zusammenfallen, verleiht dies einer Dorfgemeinschaft eine besonders starke Kohäsion (GUERMONPREZ 1984: 95).
Kennzeichen der Grundbesitzverfassung vieler Bergdörfer - wie auch anderer indonesischer Dörfer der Frühzeit - war der kommunale Landbesitz, der adatgemäß parzellenweise und manchmal jährlich rotierend Familienvorstehern ins Nutzrecht abgetreten wurde. ${ }^{2}$ Die Nutzflächen waren so bemessen, daß damit die Selbstversorgung einer Familie gesichert werden konnte und bescheidene Überschüsse zur Redistribution anläßlich von Gemeinschaftsritualen gewährleistet waren. Weideland der dörflichen Rechtsgemeinschaft wurde kollektiv genutzt. Der erwirtschaftete Surplus war mit Ausnahme des in mancher Hinsicht speziellen Agadorfes Tenganan (Regentschaft Karangasem) gering und der egalitären Ideologie der aus der Sicht des Adels ausschließlich aus Angehörigen der vierten Kaste (sudra) zusammengesetzten Dorfbevölkerung wegen auch nicht erstrebenswert. Ansehen wurde weniger über die Akkumulierung von Gütern als vielmehr über rituellen Status gewonnen.

Falls in ein und demselben Dorf Personen leben, die verschiedenen agnatischen Verwandtschaftsgruppen angehören, ergeben sich dadurch horizontale - und teilweise auch vertikale - Abgrenzungen. Denn Mitglieder einer gemeinsamen Abstammungsgruppe (soroh) fühlen sich einander deshalb verbunden, weil sie "gleicher Art" sind, d. h. ihre Herkunft auf den gemeinsamen - wirklichen oder fiktiven - Ahnvater als Ausgangspunkt (kawitan) zurückführen. Die Perzeption von Verschiedenheit beruht somit auf der Singularität der diversen Ahnenabstammungen. ${ }^{3}$ Im Gegensatz zum im Süden verbreiteten Kastensystem (oder kastenähnlichen System), bei dem die verschiedenen Abstammungsgruppen aufgrund der unterschiedlichen Wertung von Gesellschaftspositionen der diversen vergotteten Urahnen (des «Ursprungs") strikte hierarchisiert sind, bestehen zwischen den soroh von sudra - so auch in Agadörfern - geringere soziale Rangstufenunterschiede, wie auch Titelgruppen weniger häufig anzutreffen sind. Wichtig ist jedenfalls, daß der Bezug auf den gemeinsamen Ausgangspunkt die Angehörigen ein und derselben soroh sowohl dank der Abstammung vom gleichen Gründervater «verwandtschaftlich" als auch - räumlich betrachtet - durch den gleichen Ursprungsort verbindet. Das kollektive Zusammenwirken von Abstammungsgruppen kommt denn auch hauptsächlich in der Vorbereitung und Durchführung von Feiern im Ursprungstempel zum Ausdruck. (GUERMONPREZ 1984: 95, 393 f., 396).

Analog zu Bodagemeinschaften der Sasak auf Lombok (LEEMANN 1989: 9 f.) ist die Struktur der bali aga-Dorforganisation gerontokratisch. Innerhalb seines Territoriums sorgt ein Ältestenrat für Recht und Ordnung, während eng mit diesem zusammenarbeitende Dorfpriester (pamangku) über die Einhaltung religiöser Verpflichtungen wachen und als Mittler zwischen dem Diesseits und übernatürlichen Kräften wirksam sind. Der aus der verheirateten Dorfbevölkerung zusammengesetzten «Dorfversammlung» steht ein Ältestenrat vor. Versterben Mitglieder des Ältestenrates, rutschen gemäß dem Altersprinzip nächstjüngere Verheiratete nach. Visuell kommt 
dies u. a. bei der Sitzordnung im Versammlungspavillon (bale agung) zum Ausdruck, wo sich nicht nur die Dorfvertreter versammeln, sondern auch die Dorfgötter zugegen sind und wo eng miteinander verknüpfte weltliche und religiöse Dorfbelange beraten werden. Weiteres Charakteristikum von Agadörfern ist deren duale Strukturierung, die dem balinesischen Weltbild der Ausbalancierung von Gegensätzen Rechnung trägt und die Polarität uranischer und chthonischer Kräfte zu harmonisieren strebt. Solche Gegensatzpaare sind beispielsweise männlich (purusa) vs. weiblich (pradana), landeinwärts (kaja) vs. meerwärts (kelod), Richtung Sonnenaufgang (kangin) vs. Sonnenuntergang (kauh), nach oben (ke luhur) vs. nach unten (ke teben) usw. (LEEMANN 1976: 27-65).

In den Augen von Tiefland-Balinesen - vor allem aber von Angehörigen des Adels - werden bali aga als rückständig, hinterwäldlerisch und dumm ${ }^{4}$ eingestuft, seien sie doch keine "Menschen von Majapahit» (wong Majapahit). Die generelle Geringschätzung autochthoner balinesischer Werte geht beispielsweise aus der "wong Majapahit»-Version der Legende über den frühbalinesischen Lokalkönig von Pejeng (einem Dorf in der Übergangszone von der Ebene ins Bergland) hervor. Sie belegt, daß im Gedankengut vieler Balinesen die Vormajapahit-Zeit als dämonisches Mittelalter gilt und daß das Heil und die "Neuzeit» erst mit der Ankunft des legendären Majapahit-Ministers und Heerführers Gajah Mada beginnt. So wird der letzte König von Pejeng, nämlich Dalem Bedau$l u^{5}$, als halbdämonischer Herrscher mit dem Kopf eines Ebers und dem Körper eines Menschen geschildert. Die magischen Kräfte des Dalem seien so ausgeprägt gewesen, daß - in Meditation versunken - sein Haupt in den Himmel aufsteigen konnte. Eines Tages sei sein Minister über die lange «Kopflosigkeit» des Königs dermaßen beunruhigt gewesen, daß er dem erstbesten vorbeitrottenden Tier - einem Eber - das Haupt abgeschlagen und dieses dem Rumpf des Herrschers aufgesetzt habe. Der über sein unvorteilhaftes Aussehen entsetzte Dalem hätte sich von nun an zurückgezogen und allen seinen Untertanen verboten, sein Gesicht zu betrachten. Gajah Mada aber hätte vom schweineköpfigen König von Pejeng vernommen und sich über das Aussehen des sonderbaren Herrschers ins Bild setzen wollen. Es sei Gajah Mada gelungen, die Gunst des balinesischen Königs zu gewinnen. Beim Zusammentreffen mit dem Dalem sei dem Majapahit-Minister zugestanden worden, Speis und Trank für das gemeinsame Mahl zu bestimmen. Der schlaue Javane hätte sich für trockenen Reis, kangkung (Ipomoea reptans) und Wasser aus einem Krug mit langem Schnabel entschieden - alles, was nur mit nach hinten geneigtem Kopf gegessen bzw. getrunken werden kann. So sei es unmöglich gewesen, daß sich der schweineköpfige König während der Mahlzeit vor seinem javanischen Gast hätte verbergen können. Voller Scham und Wut über die List seines Gegenübers sei der Dalem Bedaulu darauf in Flammen aufgegangen. Bis heute ist der dämonische Lokalkönig mit dem Kopf eines Ebers jedem Balinesen vertraut, wird er doch in Maskenschauspielen (topeng) als - auch farblich - dunkler Herrscher dem strahlend weißen und weisen Gajah Mada gegenübergestellt.

\section{Zur Gesellschaftsorganisation der im süd- balinesischen Tiefland seßhaften Bevölkerung}

\subsection{Hindujavanische Beeinflussung}

Die balinesische Tieflandkultur basiert seit mindestens 1000 Jahren auf der Naßreisökonomie, wobei der Grundsatz gilt, daß der Boden entweder in Individualbesitz oder im Besitz einer korporativen Gruppe ist, das Wasser aber ein Kollektivgut darstellt. In dieser Gunstzone befinden sich die Paläste der Adeligen. Landeinwärts nimmt sowohl der Anteil des Adels gemessen an der Gesamtzahl der dortigen Bevölkerung ab, wie auch seine ökonomische, kulturelle und politische Bedeutung geringer wird. Balinesische Könige und andere Adelige führen ihren Ursprung und ihre Zivilisation auf die MajapahitDynastie zurück. ${ }^{6}$ So soll Mitte des 14. Jahrhunderts, zur Zeit des Majapahit-Königs Sri Hayam Wuruk, der legendäre Premierminister und Heerführer Gajah Mada Bali erobert und zu einer Provinz seines großindonesischen Reiches gemacht haben. Wie wirksam die politische Kontrolle von Majapahit über das indonesische Inselreich auch immer gewesen sein mag, ist doch mindestens der sozio-kulturelle Einfluß dieser Periode unbestritten. Denn dadurch wurde insbesondere Bali mit dem Gedankengut der hindujavanischen Sozialorganisation und des Staatsaufbaus vertraut. Zahlreiche kulturelle Innovationen wurden in der Folge absorbiert. Genereller Fokus des balinesischen Adels ist weniger Samprangan, wo der erste Palast (jav.: kraton; bal.: puri) errichtet worden war, als vielmehr Gelgel, in das Nachkommen des javanischen Susuhan Kapakisan noch vor Ende des 14. Jahrhunderts ihren puri verlegt hatten (HANNA 1976: 3). Es scheint erwiesen, daß nur gerade während der sogenannten Gelgel-Periode das gesamte Südbali unter der Oberhoheit eines einzigen, zentralen Herrschers politisch geeint war. In der zweiten Hälfte des 17. Jahrhunderts wurde Klungkung zum Sitz des Dewa Agung, ein Titel, der auf den ranghöchsten Satriahof auf Bali verweist (SWELLENGREBEL 1984: 22).

\subsection{Balinesisches Kastensystem}

Vor allem im Tiefland von Südbali schaffte der hindujavanische Einfluß neue Formen der Statusrivalität. Hier, wo die agrarische Produktivität entscheidend größer ist als im Berggebiet, weiteten sich die sozialen Bezugssysteme über das Dorf hinaus aus. Von den höfischen Zentren, vor allem von Gelgel aus, diffundierten Ideologien hierarchisierter Abstammungsgruppen, die gewisse Gemeinsamkeiten mit dem indischen Kastensystem aufweisen. Die vier Hauptkategorien der balinesischen Kasten werden kollektiv als caturwangsa (vier Kasten) bezeichnet, wobei die ersten drei Kasten (diejenigen des Adels) triwangsa genannt werden: 


\begin{tabular}{|c|c|c|c|}
\hline $\begin{array}{l}\text { caturwangsa } \\
\text { (Gesamtheit } \\
\text { aller Balinesen) }\end{array}$ & $\begin{array}{l}\text { triwangsa } \\
\text { commoners }\end{array}$ & $\begin{array}{l}\text { KASTEN } \\
\text { brahmana } \\
\text { satria } \\
\text { wesia } \\
\text { sudra }\end{array}$ & $\begin{array}{l}\text { TITEL } \\
\text { Ida } \\
\text { Dewa Agung, Dewa Gede, } \\
\text { I Dewa, Anak Agung, } \\
\text { Cokorda, I Gusti usw. } \\
\text { Gusti } \\
\text { (Innerhalb der sudra be- } \\
\text { anspruchen die Titelgruppen } \\
\text { der Pande, Pasek, Pulosari } \\
\text { Bandesa, Sengguhu usw. } \\
\text { einen höheren Status als } \\
\text { "gewöhnliche" commoners). }\end{array}$ \\
\hline
\end{tabular}

Der Rückschluß von Titeln auf die Kastenzugehörigkeit fällt vor allem bei den I Gusti und den Gusti nicht immer leicht und führt unter rivalisierenden Titelgruppen zu Kontroversen. So wird der Titel I Gusti entweder Personen der Brahmanenkaste (so im Falle von I Gusti Ngurah Sidemen und von I Gusti Dauh) oder dann der Herrscherkaste zugesprochen. Gemäß Interpretation balinesischer Lontargelehrter sollen unter den hohen Offizieren von Gajah Madas Heer Javaner gewesen sein, die nach der Eroberung Balis zu einflußreichen Beamten am Hof von Gelgel erhoben wurden. In dieser Frühphase wurde Ministern (patih) der Satriatitel Rakrian Apatih oder Krian Apatih, später I Gusti zuerkannt. Daß selbst der ranghöchste satria und heute noch lebende Enkel des letzten balinesischen Königs von Lombok, nämlich Ratu Agung I Gusti Agung Gede Jelantik Teges, die Bezeichnung I Gusti Agung in seinen Titel einschließt, ist Beleg für den hohen Status bestimmter I Gusti (die von vielen fälschlicherweise den wesia zugeordnet werden). Auch die gusti, die der Wesiakaste angehören, führen ihre Herkunft auf verdienstvolle Begleiter von Gajah Mada zurück. So sollen etwa den Gajah Mada-Gefolgsleuten Tan Mundur, Tan Kaur und Tan Kober der Titel Gusti (ohne I!) zuerkannt worden sein. Der auf einen hohen Rang innerhalb der $\mathrm{Sa}$ triakaste verweisende Titel Dewa ist auf Nachkommen des Dewa Raja von Klungkung beschränkt, dessen Vorfahren gemäß Klungkung-Version ursprünglich der javanischen Brahmanenkaste angehört hatten. Als ein Angehöriger dieser Abstammungsgruppe die Funktion des Königs von Klungkung übernommen habe, soll er von der Brahmanen- in die Herrscherkaste (satria) gewechselt haben, wobei ihm und seinen Thronfolgern der ehrenvolle Titel I Dewa Agung, seinen Nachkommen ohne Königsfunktion der Titel I Dewa zugesprochen worden sei.

Aus diesen Beispielen geht hervor, daß auch innerhalb einer Kaste wiederum ausgeprägte Statusnuancen bestehen. Dies gilt auch für Brahmanen. Angehörige der brahmana paksa buda führen ihren Ursprung auf den javanischen Priester Dang Hyang Astapaka zurück, so beispielsweise die Brahmanen von Budakeling, Wanasari und Batuan. Brahmanen siwaistischer Prägung ihrerseits sehen als ihren Stammvater den jüngeren Bruder von Dyang Hyang Astapaka, nämlich Dang Hyang Nirartha (synonym dazu Dang Hyang Dwijendra oder Ida Batara Padanda Sakti Wau Rauh)? der im 15./16. Jahrhundert auf seiner legendären Missionierungsreise (dharma yatra) durch Bali, Lombok und Sumbawa den Hinduismus entscheidend revitalisiert und zahlreiche Tempel gegründet haben soll.

Balinesen erklären die Schichtung der Brahmanenkaste aufgrund der unterschiedlichen Gesellschaftspositionen der fünf Ehefrauen des missionierenden Hindupriesters, die auf den Status ihrer Nachkommen abfärbten. So soll seine erste Gemahlin eine Priestertochter aus dem javanischen Königreich Daha (Kadiri) gewesen sein, die ihm die Tochter Dalem Melanting und den Sohn Dang Hyang Wiraga Sandhi (syn.: Batara Padanda Sakti Kemenuh) geschenkt habe. Letzterer sei zum Begründer der Linie der Brahmana Kemenuh geworden, die auf Bali höchstes Ansehen genießt. Als zweite Frau habe Dang Hyang Nirartha eine Priestertochter aus dem ostjavanischen Königreich Pasuruhan geehelicht und mit ihr die vier Kinder Padanda Sakti Kulon, Wetan, Lor und Ler gezeugt. Nachfahren aus dieser Verbindung nennen sich Brahmana Manuaba. Der Ehe mit seiner dritten Gemahlin, einer Brahmanentochter aus dem ostjavanischen Kleinkönigreich Blambangan, seien die drei Kinder Ida Padanda Keniten, Ida Sakti Telaga und Ida Padanda Isteri Rai entsprungen. Aus dieser Verbindung leitet sich die balinesische Gruppe der Brahmana Keniten ab. In der Folge sich im Dorf Mas (Gianyar, Bali) aufhaltend, hätte sich Dang Hyang Nirartha dort mit einer Tochter des angesehenen, in den Augen von wong Majapahit aber nichtadeligen Balinesen Bandesa Mas vermählt. Der aus dieser Ehe entsprossene Sohn und spätere Padanda Gede Mas ist Begründer der Linie der Brahmana Mas. Die fünfte Ehefrau mit niedrigstem Status endlich sei Ni Brit, eine Dienerin des Bandesa Mas, gewesen. Ihre zwei Söhne Ida Patapan und Ida Binduwerden als Begründer der am tiefsten eingestuften Brahmanenlinie, der sogenannten $A n$ tapan Mas, betrachtet. ${ }^{8}$

Daß im Terminus brahmana catur («vier Brahmanen[linien]») nur vier der fünf oben angesprochenen Abstammungslinien genannt werden, mag darauf beruhen, daß die Mütter aus vier verschiedenen Herkunftsregionen stammen: den oben genannten drei javanischen Königreichen Daha, Pasuruhan und Blambangan sowie 
aus dem südbalinesischen Dorf Mas. Es wäre freilich eine weitere Interpretation überprüfenswert, nämlich, ob die fünfte Ehefrau (Ni Brit) aufgrund ihres niedrigen Status nicht klassifiziert worden sei.

Wie von Adeligen sozial tiefer klassierte Balinesen durch Bezugnahme auf legendäre javanische Brahmanenpriester ihre Gesellschaftsposition anzuheben trachten, soll am Beispiel der Titelgruppe der sengguhu aufgezeigt werden. Gemäß Chronik der Titelgruppe der pasek (SUGRIWA 1990: 59-68) soll der javanische Priester Mpu Dwijaksara auf Geheiß von Gajah Mada Mitte 14. Jahrhundert nach Bali gesandt worden sein, um in mehreren Tempeln die korrekte Abwicklung der puja wali-Zeremonien zu überwachen und damit Glück und Wohlfahrt auf der Insel zu gewährleisten. Zur gleichen Zeit soll sich Sang Kulpetak, begleitet von seinem frommen Diener I Guto, nach Bali begeben haben. Dank seinem engen Kontakt mit Sang Kulpetak hätte dessen Begleiter im Verlauf der Zeit die heiligen Weden, die mantra und mudra bald ebenso gut beherrscht wie sein Herr, und I Gutos Gehabe und Auftreten hätte sich in nichts mehr von dem eines Priesters unterschieden. Auf seinen Wanderungen durch die Dörfer sei I Guto mit "unwissenden" Bauern in Kontakt gekommen, denen der javanische Glaubensverkünder Mpu Dwijaksara nur dem Namen nach bekannt gewesen sei. Deshalb hätten sie auch den Diener mit dem Brahmanenpriester verwechselt. ${ }^{9}$ In Verkennung der Person hätte ein Dorfbewohner den vermeintlichen $M p u$ Dwijaksara-der in Wirklichkeit niemand anders war als $I$ Guto! - in sein einfaches Haus geladen und ihn dort um Beistand bei einer Kulthandlung für Verstorbene gebeten. Der geschmeichelte Diener hätte darauf das Ritual ohne Fehl und Tadel, aufgrund seiner Kastenzugehörigkeit aber unberechtigterweise, geleitet. Dabei hätte ihn der erst später hinzukommende Mpu Dwijaksara ertappt und zur Rede gestellt. Nach Abklärung des Irrtums seitens des Dorfbewohners hätte der wahre Priester dem reuigen I Guto als Verwechseltem (sengguh) in Anerkennung seiner Religionskenntnisse und seines guten Willens die Erlaubnis erteilt, auf Ersuchen von Bittstellern hin auch weiterhin bestimmte Zeremonien für Verstorbene (pitra yadnya) leiten zu dürfen. Bis heute steht den Nachkommen von I Guto, den sengguhu, dieses Vorrecht zu. ${ }^{10}$ Während balinesische Adelige die sengguhu aber nur als Viertkastige in gehobener Position klassieren, wehrt sich diese Titelgruppe entschieden gegen eine Zuweisung zur Kaste der sudra.

Wesentliche Unterschiede zum Kastensystem in Indien ergeben sich aus der Tatsache, daß auf Bali die Klasse der Unberührbaren fehlt und daß - von wenigen Ausnahmen abgesehen - Erwerbstätigkeiten nicht kastengebunden sind, wie ganz allgemein weniger kastenspezifische tabu bestehen. Als auffallende Ausnahme davon sei das Amt des Brahmanenpriesters (padanda) genannt, das ausschließlich Männern der Priesterkaste offensteht. Für gläubige Balinesen basiert religiöse Andacht weniger auf individueller Reflexion oder Fürbitte als auf gefühlsmäBiger Erfahrung in der Gemeinschaft. Die Hauptaufgabe des Priesters ist die Umsetzung seines Wissens zur
Kontaktherstellung mit Gott. Das Gotteserlebnis der Gläubigenschar erfolgt somit über den höher thronenden padanda. Seine direkten Einnahmequellen sind auf den Erlös aus dem Verkauf des von ihm geweihten Wassers (tirta) beschränkt. Zudem dürfen Priester auch Geschenke für ihre religiösen Dienste von einladenden Familien und Tempelkongregationen entgegennehmen. Als "Reinste» unter den Lebenden sind ihnen manuelle («unreine») Berufstätigkeiten untersagt. Ebenso unterliegen sie als einzige Gruppe restriktiven Essensvorschriften. So ist ihnen beispielsweise der Genuß von Hühnerfleisch, aber nicht von Entenfleisch, untersagt." Die von einladenden Familien und Kongregationen gereichten Speisen müssen auf neuen Tellern dargeboten werden. Sollten Gastgeber diese Voraussetzungen nicht erfüllen können, wird dem padanda das Essen in frischen Bananenblättern gereicht.

Padanda kamen nebst religiösen ursprünglich auch richterliche Funktionen zu. Ebenso wirkten sie als königliche Berater. Ihre Vorzugsstellung kommt darin zum Ausdruck, daß in früheren Zeiten Brahmanenpriester nicht zum Tode verurteilt werden durften. Sie schuldeten Herrschern keine Steuern. Ihre Gegenleistung an den Raja bestand darin, für die Wohlfahrt des Königreichs zu beten und den Herrscher spirituell zu stärken.

Der Beruf der Schmiede steht ausschließlich Angehörigen der Titelgruppen der pande zu. Von anderen Balinesen als hochrangigere sudra eingestuft, geben sich Schmiede selber als Angehörige der Herrscherkaste aus und versuchen wie diese, in Stammbäumen ihre adelige Herkunft zu belegen. Der Konflikt ist so nicht nur mit den satria, sondern auch mit den brahmana vorprogrammiert, da pande entgegen der Hindudoktrin Priester aus ihren eigenen Reihen stellen und Wasser selbst zu tirta weihen. Türme (bade), auf denen Schmiede Leichen zur Kremationsstätte tragen, weisen - wie beispielsweise im Dorf Marga - sieben Dächer auf, gleich viele, wie es andernorts nur satria zusteht. Im erwähnten Dorf werden verstorbene pande in einem Sarg in Form eines schwarzen Bullen kremiert, dem Sargtyp, der in der Regel für nicht das Priesteramt ausübende brahmanabestimmt ist.

$\mathrm{Zu}$ den wenigen Berufen, die als unrein taxiert werden und dementsprechend geringes Ansehen genießen, zählen Metzger und Stoffärber. Es sind die untersten sudra, die ihren Lebensunterhalt durch diese Erwerbstätigkeiten bestreiten.

Das balinesische Kastensystem ist freilich weniger starr als dies auf den ersten Blick erscheinen mag. So soll - wie bereits oben erwähnt - die ranghöchste balinesische Königsfamilie, nämlich diejenige von Klungkung, ursprünglich der Brahmanenkaste angehört und erst nach Übernahme der Herrscherfunktion in die Satriakaste gewechselt haben. Die Identifikation der balinesischen Elite mit dem hindujavanischen Hochadel und dessen Gefolgsleuten scheint erst ab rund 250 Jahren nach der «Eroberung» durch Gajah Mada entwickelt worden zu sein (SCHULTE NORDHOLT 1986: 12 f.). Mit fiktiven Genealogien (silsilah) versuchte der balinesische Adel, seine di- 
rekte Abstammung auf hochrangige javanische Persönlichkeiten zurückzuführen und - wie etwa im Fall der Königsfamilie von Karangasem - mit einem Gottessohn (Batara Alit Sakti) aufzuwerten. Bis heute sind solche Stammbäume konfliktgeladener Diskussionsstoff unter rivalisierenden Herrscherhäusern. Im Extremfall werden mißliebige Satriageschlechter sogar als machtgierige Emporkömmlinge aus der Gruppe der commoners diskriminiert (vgl. dazu die Kontroversen um den Ursprung der Pamecutan-Dynastie in Badung). Eine gewisse vertikale Mobilität innerhalb der balinesischen Gesellschaft lässt sich daran belegen, daß beispielsweise verdienstvolle Untertanen von ihrem Herrscher in den niedrigen Adel angehoben werden konnten und dann den Titel Gusti zu tragen berechtigt waren. Statusverschiebungen ergeben sich auch durch legale Heiraten balinesischer Adeliger mit Frauen aus der Sudrakaste. Solchen Ehen entspringenden Kindern fällt automatisch der Status ihres Vaters zu, während deren Mütter weiterhin der vierten Kaste angehören, wenn auch in gehobenerer Position als außerhalb des Palastes wohnhafte commoners.

Die Präsenz der wangsa wird in der Vielfalt hierarchischer Gruppen augenfällig, deren Interaktionen bestimmten Regeln und Praktiken unterliegen. Angehörige der triwangsa (rund 7\% aller Balinesen), aber auch bestimmte höher eingestufte Sudragruppierungen legen Wert darauf, daß die an die entsprechenden Gesellschaftspositionen gebundenen Umgangsformen beachtet werden. Im Gegensatz zu Indien beschränken sich balinesische Kastenvorschriften in erster Linie auf fixierte Anstandsregeln. Mangel an guten Manieren und Formen ist für Balinesen ein gravierender Vorwurf. Die Besorgnis um Einhaltung korrekter Umgangsformen bei bevorstehenden Interaktionen kann die Partner dann psychisch verunsichern und sich hemmend auf ihr Handeln auswirken, wenn sich Begegnende beispielsweise über ihren Statusunterschied nicht im klaren sind. Dieses charakteristisch indonesische Verunsicherungsphänomen wird mit dem malayischen Wort "malu» ausgedrückt, dessen Sinngehalt mit «beschämt», "schüchtern», "zaghaft» oder «ehrfurchtsvoll» nur unvollkommen erfaßt wird.

Ein in der Hierarchie tiefer Stehender hat darauf zu achten, daß er im Umgang mit Höhergestellten seine ihm zukommende Rolle spielt und seine Position demonstriert. Grüßt ein sudra einen Adeligen, hat er zur Respektbezeugung seinen Kopf zu senken. Pavillons in Palästen weisen gestufte Plattformen auf, die es erlauben, auf statuskonformer Höhe zu gehen oder zu sitzen. In konservativen Kreisen des Hochadels ist es bis heute üblich, daß - häufig aus dem Sklavenstand hervorgegangene - Leibdiener sich ihren Gebietern kriechend nähern und dabei die Stirn auf den Boden pressen. Schon den Staub der Füße des Königs erblicken zu dürfen, ist eine Ehre, geschweige denn die Wahrnehmung der ganzen Größe der Person! Dieser Sinngehalt kommt in der früher üblichen Höflichkeitsadresse an einen König zum Ausdruck, der mit dem Titel Bukpadan Cokor I Dewa angesprochen wurde. ${ }^{12}$ Unterwürfig haben sich gemäß adat von Karangasem uneheliche Kinder eines Adeligen und einer Sudramutter gegenüber ihrem Vater zu verhalten. Solange sie im Palast leben, fällt ihnen als sogenannte astra die Rolle eines demütigen Dieners zu. Uneheliche Kinder beiderseits adeliger Eltern hingegen werden voll in die väterliche Familie integriert. Bei Gemeinschaftsmahlzeiten beginnt der Ranghöchste mit dem Essen, und keinem ist es gestattet, die Tafelrunde zu verlassen, bevor der Höchststehende die Mahlzeit als beendet erklärt hat. Diese plausible Anstandsregel schuf in der Vorphase des Massentourismus unerwartete Probleme. So erinnere ich mich, wie 1967 balinesische Lokalführer freundlicherweise von Touristen zur gemeinsamen Mahlzeit eingeladen wurden. Doch wer sollte zuerst bedient werden und die Tafelrunde zum Essen auffordern: der adelige balinesische Angestellte oder der zahlende Gast, der in den Augen der Balinesen als Außenstehender ein sudra ist? Noch bis um 1970 wurde die Nichtvereinbarkeit dieser "Östlichen" und "westlichen" Anstandsregel elegant umgangen, indem balinesische Fremdenführer wohl die Einladung gerne annahmen, aber in einem separaten Raum aßen und Angehörige der triwangsa dort denn auch wirklich zuerst bedient wurden. Weitere Verunsicherungen ergaben sich aus dem Umstand, daß unterschiedlichen Kasten angehörende Angestellte einheimischer Reisebüros und Hotels unbesehen von der gesellschaftlichen Position über- oder untergeordnete Funktionen wahrzunehmen hatten.

Hand in Hand mit der Akzeptierung des Kastensystems ging die Einführung adäquater Sprachebenen, die es in der Konversation zwischen Angehörigen unterschiedlicher Kasten und Kastenabstufungen zu befolgen gilt. Treffen sich zwei Balinesen, die sich nicht kennen, sprechen sie vorerst in madya, der "mittleren Sprache», die als Kompromißsprache zwischen kasar («niedrige Sprache») und alus (Hochsprache des Adels) aufgefaßt werden kann. Im konservativen Ostbali nimmt man in solchen Fällen die Konversation in alus auf. Sobald dann die unterschiedliche Kastenzugehörigkeit der Gesprächspartner offensichtlich geworden ist, erinnert sich ein sudra der Grundregel, daß er als Viertkastiger zur Respektbezeugung in alus weitersprechen muß, während der Adelige sich in kasar an sein Gegenüber wendet. Im Gegensatz zur balinesischen Kasarsprache ist alus nuanciert und enthält raffinierte poetische Umschreibungen. Der Anstand gebietet, daß eine über sich selbst redende Person sich nicht der Hochkastensprache bedient. Hingegen sprechen wohlerzogene Balinesen ihre Eltern und Alte zur Respektbezeugung in alus an. ${ }^{13}$ Innerhalb des Adels drückt man sich je nach Region in kasar oder madya aus, außer man richte das Wort an seine Eltern, ältere Brüder oder an Priester. Der in alus Angesprochene antwortet in der Niedrigkastensprache (kasar). Während im urbanisierten Denpasar und in Nordbali fast ausschließlich kasar und madya gesprochen wird, verwenden Bewohner der konservativeren Regentschaft Karangasem hauptsächlich madya und alus. Unterrichtssprache in den Schulen der rasch wachsenden Provinzhauptstadt 
Denpasar ist kasar, in Ostbali madya. Priester, Lontarleser, Puppenspieler (dalang) und andere klassisch geschulte Balinesen beherrschen zudem Altbalinesisch, Altjavanisch (kawi), gelegentlich auch Sanskrit. Auf allen Schulstufen wird die indonesische Nationalsprache $\mathrm{Ba}$ hasa Indonesia gelehrt. Als weitere Idiome lernen die Schüler in erster Linie Englisch, manchmal zusätzlich Japanisch, Französisch oder Deutsch. Nur noch ältere, von Niederländern geschulte Balinesen sprechen Holländisch.

\subsection{Weitere Gruppenzugehörigkeiten}

Wenn auch die balinesische Gesellschaft sowohl durch Kasten als auch durch kasteninterne Rangierungen deutlich stratifiziert ist, darf nicht außer acht gelassen werden, daß Balinesen nebst ihrer Zugehörigkeit zu agnatischen Gruppen mit unterschiedlicher Wertung aufgrund ihres Ursprungs ebenso Mitglieder zahlreicher anderer, kastenübergreifender Gruppen sind. Während die Geschlossenheit von Abstammungsgruppen vor allem bei Gemeinschaftsritualen in ihrem Ursprungstempel zum Ausdruck kommt, spielen die anderen Gruppierungen im Alltag eine wesentlichere Rolle. Im vorkolonialen Bali war der Lebensbereich von commoners hauptsächlich auf ihr Dorf (desa) und dessen Untereinheiten (banjar) beschränkt, während das Netzwerk des Adels (Priester, Herrscher) überdörflich organisiert war und Großregionen umfaßte. Die Einbindung der innerhalb eines Königreiches gelegenen Dorfgemeinschaften erfolgte über königliche Beamte, die entweder als in der Hauptstadt niedergelassene oder durch im Dorf selbst seßhafte Adelige die Implementierung königlicher Edikte zu überwachen hatten. Da die Machtposition eines Herrscherhauses nicht zuletzt mit der Größe ihrer commoner-Gefolgschaft korrelierte, richtete sich das Bestreben der Herrschenden darauf, ihren Einflußbereich auf Kosten ihrer Rivalen auszuweiten. Dieses Ränkespiels rivalisierender Potentaten waren sich die commoners wohl bewußt. Letztere wußten diese Situation zu ihren Gunsten zu nutzen, indem sie dem Herrscher Gehorsam zu schulden und Tribute zu entrichten versprachen, der ihnen die besseren Konditionen zu gewährleisten bereit war (H. GEERTZ und C. GEERTZ 1975: 5 ff.). Ein solcher Wechsel von einem Herrscher zu einem anderen wird durch den balinesischen Begriff matilas ausgedrückt.

Da die Gesellschaftsstruktur der Balinesen untrennbar mit religiösen Konzepten und Zeremonien verbunden ist, sind die Aktivitäten der diversen korporativen Gruppen auf spezifische Tempel konzentriert. Dabei setzen sich die verschiedenen Tempelkongregationen aus unterschiedlichen Mitgliedern zusammen. Dies kann beispielsweise anhand der von H. GEERTZ und C. GEERTZ (1975: 14 ff.) erwähnten Kategorisierung in die vor allem im südlichen Tiefland vorzufindenden Bereiche Dorf, banjar, Bewässerungsgemeinschaft, Titelgruppe, Verwandschaftsgruppe, "Clubs» und um Brahmanenpriester konzentrierte Gruppen belegt werden. Im folgenden soll das Augenmerk vorerst auf die Bedeutung des Dorfes und des banjar als korporative Gruppen gerichtet werden. Nebst der Familie und der Verwandtschaftsgruppe ist der banjar die wichtigste Bezugsgruppe im Leben von commoners. Banjarsind einerseits klar abgegrenzte Territorien innerhalb eines Dorfes (desa adat). In dieser Bedeutung sind banjar Wohnviertel, gewissermaßen die Wohnadresse von Dorfbewohnern. Andererseits bilden banjar als Nachbarschaften sozio-politische Gruppierungen, denen im Dorfleben bestimmte Pflichten, Rechte und Befugnisse zufallen. In manchen Fällen ist die Zugehörigkeit zu einem banjar im Sinne einer sozio-politischen Einheit nicht mit der banjar-Wohnadresse identisch (SCHAAREMAN 1986: 45 f.). Um der angesprochenen Doppeldeutigkeit des Begriffes zu entgehen, soll im folgenden banjar in seiner Bedeutung als sozio-politische Gruppierung verstanden werden.

\subsubsection{Banjar-Gemeinschaft}

Die Mitgliedschaft im banjar setzt sich aus erwachsenen Männern zusammen, die sich über einen weiblichen Partner - in der Regel eine Ehefrau, manchmal aber auch eine Schwester, Mutter oder Tochter - ausweisen können. Diese Regelung gilt nicht zuletzt deshalb, da einem banjar auch geschlechtsspezifische Aufgaben zufallen. Dazu zählen beispielsweise die Herstellung und Darbietung von Opfergaben für Tempelzeremonien und für Banjarsitzungen usw., Aufgaben, die Frauen vorbehalten sind. Verwitwete, Ledige und manchmal auch Neuzuzüger haben nicht allen Banjarbestimmungen nachzukommen, sondern sind lediglich "halbverpflichtet" (setengah ayahan). Alte und Gebrechliche schließlich können sich gänzlich zurückziehen und sich durch ihre verheirateten Söhne vertreten lassen.

$\mathrm{Zu}$ den wesentlichen Pflichten eines banjar zählen die Aufrechterhaltung und Wiederherstellung der rituellen Reinheit des desa adat. Jeder banjar hat spezifische Arbeiten und rituelle Aufgaben zugunsten der Dorftempel zu erfüllen. Daraus kann geschlossen werden, daß das Dorf als religiös-magische Autorität der säkularen des banjar übergeordnet ist. Solange aber die Reinheit eines desa als Ganzes gewährleistet ist, werden die Eigenbefugnisse eines banjar nicht tangiert und die Eigenständigkeit dieser Gruppierung betont. Banjaraktivitäten können sehr realitätsbezogen sein, so etwa, wenn es gilt, im Gemeinschaftswerk Wege auszubessern oder das banjareigene Versammlungshaus (bale banjar) zu restaurieren. Der banjar ist generell für seine öffentlichen Einrichtungen zuständig und - mehr noch - für die öffentliche Wohlfahrt verantwortlich, die nebst materieller Unterstützung auch Beistand in rituellen Belangen erfordert. So sind bei der Vorbereitung und Durchführung von rites de passage - wie beispielsweise bei der Zahnfeilzeremonie (vgl. GH 1992/4) - nicht nur Familienangehörige und Verwandte, sondern auch Banjarmitglieder zur Mitwirkung aufgefordert. Frauen bereiten in zeitaufwendiger Arbeit Opfergaben für die Götter (banten) oder zur Beschwichtigung von Dämonen (caru) vor. Alte mögen die 
Zeremonie mit Gesängen begleiten, andere an einem rituellen Puppenspiel bei Tageslicht (wayang lemah) mitwirken. Noch augenfälliger und zeitraubender ist die für Banjarmitglieder verpflichtende Mitwirkung bei der Beerdigung und Kremation Verstorbener. ${ }^{14}$ So sind die Männer angehalten, den kastenkonformen Sarg und dessen Tragbahre aus Bambus zu erstellen sowie einen reich geschmückten Leichentragturm (bade) zu errichten. Gemäß dem adat der Schmiede von Marga ist es Pflicht der Burschenschaft, den leeren Sarg zur Einäscherungsstätte zu tragen, während die verheirateten Männer des banjar den bade, auf dem der Leichnam ruht, zum dorfeigenen Kremationsplatz bringen. Die restlichen Banjarangehörigen begleiten den Leichenzug. Auf der Einäscherungsstätte angelangt, wird der Tote in den Sarg überführt, unter dem vom banjar beschafftes Holz mit einer Fackel oder einem Brennglas entzündet wird. Für die eigentliche Einäscherung verbleiben nur die Angehörigen auf dem Platz sowie einige Männer, denen die vom banjarzugesprochene Aufgabe zufällt, dafür zu sorgen, daß der über den Fallboden des Sarges ins Feuer gleitende Leichnam vollständig eingeäschert wird.

Eine weitere Aufgabe des banjar liegt in der sozialen Kontrolle und der Überwachung des adatgemäßen Handelns seiner Mitglieder. So ziehen in den banjar der großen Gemeinde Sanur beispielsweise mit Sakralspießen (tumbak) bewaffnete Männer am Übergangstag vom alten zum neuen Saka-Jahr - am hari raya nyepi- durch die Wohnquartiere und überwachen die verbindliche Einhaltung der Ruhebestimmungen und des Verbotes des Feuerentfachens. ${ }^{15}$ Sollten Banjarmitglieder die adatgemäßen Verhaltensregeln nicht befolgen, steht dem banjar das Recht zur Ergreifung von Sanktionen zu, und Bußen können ausgesprochen werden. Besonders gravierende Vergehen gegen die Banjarregeln werden durch Verstoßung des Missetäters geahndet. Dies bedeutet für den Betreffenden nicht nur den Verzicht auf seinen Anteil am Banjarbesitz und den Entzug seiner Wohnlandparzelle, sondern in letzter Konsequenz auch den Verlust des Rechts auf eine adatgemäße Bestattung auf dem Dorffriedhof und die nachfolgende Kremation. Er ist zum Außenseiter geworden und hat nun - ohne Absicherung durch die Banjargemeinschaft - eine neue Existenz fernab seines Heimatdorfes zu suchen.

Einmal im balinesischen Monat zu 35 Tagen treffen sich die Banjarmitglieder zur Sitzung im bale banjar oderfalls kein solcher vorhanden ist - im Ursprungstempel (pura puseh). Dem banjar steht ein für eine bestimmte Zeitperiode gewählter klian banjar vor, der in seiner Arbeit von verschiedenen Assistenten unterstützt wird. Unbesehen von seiner Kastenzugehörigkeit ist jedes Mitglied gleichberechtigt. Selbst der Banjarvorsteher besitzt keine besonderen Machtbefugnisse, denn er ist lediglich dafür besorgt, daß den Beschlüssen der Versammlung Nachachtung verschafft wird. So überwacht er beispielsweise die Durchführung von Gemeinschaftsarbeiten und ist für das Einziehen des Banjarbeitrages zuständig (H. GEERTZ und C. GEERTZ 1975: 17 f.). Beschlüsse der Ban- jarversammlung werden gemäß dem altindonesischen Grundprinzip des musyawarah untuk mufakat, d.h. der Diskussion zur Konsensfindung, einstimmig gefaßt. In der Regel ist die Entscheidungsfindung bereits vor der Versammlung durch ausgiebiges Lobbying vorbereitet worden, so daß sich der Sitzungsverlauf in gediegener Würde und Ruhe abwickelt.

Manche banjar verfügen über ein ansehnliches Vermögen. Dazu zählen Immobilien wie der bale banjar und die Hahnenkampfhalle (wantilan). Bale banjarerfüllen nebst ihrer Funktion als Versammlungshaus auch eine Rolle als informelle Treffpunkte, wo sich die Männer nach getaner Arbeit bei einem Umtrunk und Snack zum Palaver treffen. Falls erst wenige Bewohner über einen eigenen TV-Apparat verfügen, haben wohlhabendere banjarin ihrem Versammlungshaus einen Fernseher stehen. Tagsüber mögen Kinder auf der Terrasse Tischtennis spielen oder auf banjareigenen Gamelaninstrumenten üben. Bis zur rigorosen Limitierung der Hahnenkämpfe (gocekan) durch einen Gesetzeserlaß der indonesischen Zentralregierung bezüglich des Verbots von Glücksspielen Ende der siebziger Jahre flossen manchen Banjarkassen durch verbindliche Wettgeldabgaben bedeutende finanzielle Mittel zu. Des weiteren wird die Banjarkasse durch wenn auch bescheidene - monatliche Mitgliederbeiträge geäufnet. Das Geld mag in banjareigene Wirtschaftsaktivitäten investiert werden. Gut geschützt werden Barongund Rangdamasken aufbewahrt, deren magische Ausstrahlungskräfte einmal im 210-Tage-Jahr von der ganzen Banjargemeinschaft reaktiviert werden. Manche banjar besitzen eine eigene Reisscheune, in der "Reissteuern» von Mitgliedern bzw. Reisgarben von banjareigenen Feldern aufbewahrt und anläßlich zeremonieller Anlässe teilweise redistributiert werden.

Anders als ein klian banjar adat, der in seinem Wirkungskreis für Adatbelange zuständig ist, fällt einem klian banjar dinas die Verantwortung über die Implementierung von Erlassen der regionalen bzw. nationalen Regierung zu.

\subsubsection{Dorfgemeinschaft}

Das Wort "desa» (Dorf) hat in seinem territorialen Bezug mehrere Konnotationen. So kann in Europa "Dorf" im engeren Sinne sich auf den überbauten und bewohnten Kernteil des Dorfterritoriums beschränken, im weiteren Sinn aber auch die bebaute und unbebaute Fläche innerhalb der Dorfgrenzen umfassen. Ähnlich auf Bali und anderen indonesischen Inseln: Einerseits handelt es sich beim desa um den innerhalb eines ursrpünglich durch eine Mauer oder einen Zaun begrenzten Teil des Dorflandes, auf dem die Familienhöfe (pakarangan) und die Dorftempel stehen, andererseits um das gesamte Territorium, über welches die Dorfgemeinschaft als korporative Gruppe legale Rechte beansprucht. Im folgenden soll der Begriff "desa adat" in seiner umfassenderen Bedeutung verstanden werden, d.h. als das Dorfterritorium mit seinen Familienhöfen, Tempeln, landwirtschaftlichen Nutz- 
flächen verschiedenster Art und eventuell nicht genutzten Landreserven. Innerhalb dieser Grenzen legen die in den heiligen Dorfsatzungen (awig-awig) enthaltenen Regeln das Verhalten der Dorfbewohner fest (SCHAAREMAN 1986: 44). Da im Gedankengut der Dorfbevölkerung das Dorfterritorium letztlich als Besitz der Götter betrachtet wird, der temporär von Menschen verwaltet und von den Familien bewirtschaftet wird, ist es primäres Ziel einer jeden Dorfgemeinschaft, als Adatrechtsgemeinschaft über die rituelle Reinheit ihres Territoriums zu wachen und diese im Falle von Störungen wiederherzustellen. Handlungen und Ereignisse, welche die Unreinheit des Dorfterritoriums und dessen Bewohner zur Folge haben, bilden Verstöße gegen das adat und beeinträchtigen als solche die kosmische Harmonie. Während die Hauptaufgaben der einem desa untergeordneten banjar in erster Linie im säkularen und sichtbaren Bereich (sekala) liegen, so zum Beispiel in der Regelung menschlicher Interaktionen und in der Erfüllung spezifischer Aufgaben für die Dorftempel, ist - wie oben angetönt - die Institution desa adat für die magisch-religiöse Domäne zuständig und für die Beziehungen zwischen den Dorfbewohnern und dem Unsichtbaren (niskala) bestimmend. Wenn das desa adat primär symbolische Funktionen hat und die Gesellschaftlichkeit definiert, ist es der banjar, der diese produziert (H. GEERTZ und C. GEERTZ 1975: 16).

Die Gruppe der Erben von Familienhöfen und zugewanderte Familienvorsteher, die vom desa adat Land für einen pakarangan zugesprochen erhalten haben, bilden die vollberechtigten Mitglieder einer Dorfgemeinschaft (krama desa) (HOBART 1980: 85, 102; GUERMONPREZ 1984: 384). Sollte ein Dorfangehöriger ohne Nachkommen (eigene oder adoptierte) sterben, fallen sowohl sein Nutzrecht auf landwirtschaftliche Parzellen auf Dorfland als auch der Boden, auf dem sein Familienhof steht, ins Dorfgut zurück. Damit kommt deutlich zum Ausdruck, daß es die Desagemeinschaft - und nicht etwa der banjar - ist, welche als korporative Gruppe das exklusive Verfügungsrecht über dorfeigenes Land besitzt (SCHAAREMAN 1986: 82). Zum desa adatgehören aber ebenso die Dorftempel, in denen sämtliche Mitglieder dorfinterner Gruppierungen ihren Göttern huldigen und ihr Weihwasser (tirta) beziehen. Als Glaubenskongregation haben die Mitglieder der Dorfgemeinschaft ihren religiösen Verpflichtungen in den genannten Tempeln nachzukommen, diese zu unterhalten und Sakralhandlungen zu organisieren.

Idealerweise umfassen die Dorftempel drei pura, die insgesamt als kahyangan tiga ("die drei Sanktuarien») bezeichnet werden. Es sind dies der pura puseh, der pura desa (syn. pura bale agung) und der pura dalem. ${ }^{16}$ Beim pura puseh ("Nabeltempel») handelt es sich um den Ursprungstempel, in dem die Dorfgemeinschaft ihre purifizierten und vergotteten Ahnen verehrt. Seiner uranischen Wertung wegen liegt er in der «göttlichen Richtung», d. h. bergwärts und/oder östlich des Dorfkerns. Im Zentrum des Dorfes - im Spielfeld irdischer und göttlicher Einflüsse also - befindet sich der pura desa. Der pura dalem endlich ist sowohl der Tempel des Todes als auch der chthonischen Mächte, wo u. a. noch nicht vollständig purifizierter Verstorbener gedacht wird. Diesem Tempel ist der Dorffriedhof (sema) und ein Kremationsplatz (setra) beigefügt. Der Wertung der Kardinalrichtungen gemäß liegt der pura dalem im meerwärts situierten und/ oder westlichen Dorfteil. Manchmal ist er vom eigentlichen Wohngebiet isoliert gelegen (vgl. Abb. 1; LEEMANN 1976: 46-48). Auffallend ist, daß der pura dalem, der Friedhof und die Einäscherungsstätte im Besitz des Dorfes sind, Beerdigungen, Kremationen und nachfolgende Purifikationszeremonien aber banjarweise durchgeführt werden, somit diese Rituale als eines der Bindeglieder zwischen den Institutionen desa und banjar wirken.

Traditioneller Vorsteher eines desa adat ist der bandesa adat. Als solcher ist er für die Innehaltung der heiligen Dorfsatzungen zuständig und verantwortlich für die Tempel, in denen die dorfspezifischen Feiern stattfinden. Bei Unklarheit in der Interpretation verhaltensspezifischer Regeln suchen die Dorfbewohner bei ihm Rat. Kei-

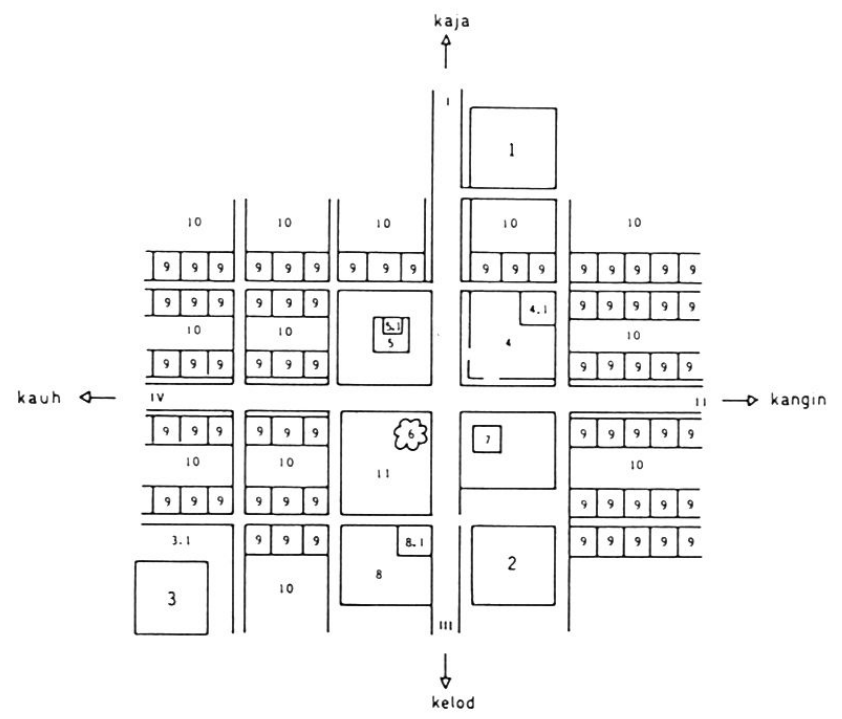

Legende:

1: Pura Puseh

2: Pura Desa (Pura Bale Agung)

3: Pura Dalem

3.1 Friedhof und Kremationsstätte

4: Puri (Palast einer Satria-Familie)

4.1 Pemrajan (Tempel einer Adelsfamilie)

5: Wantilan: offene Halle für Hahnenkämpfe und Vorstellungen

5.1 Altar für Batara Bagus Bebotoh, den Gott der Spieler 6: Waringin-Baum (Ficus benjamina)

7: Kulkul (Schlitztrommel)

8: Melanting (Marktplatz)

8.1 Pura Melanting (Altar für die Gottheit des Marktes)

9: Pakarangan (Familienhof)

10: Gärten und/oder Felder

11: Dorfplatz

I, II, III, IV: Hauptstraßen

Abb. 1 Idealtypischer Grundriß eines südbalinesischen Dorfes 
nesfalls ist die Stellung eines bandesa mit derjenigen eines Gemeindepräsidenten vergleichbar. Denn ein desa adat-Vorsteher hat keinerlei Machtbefugnisse zur Durchsetzung von rechtlichen oder politischen Entscheiden. Die Macht dazu liegt - wie früher erwähnt - in den Händen der Banjargemeinschaft.

Dorfversammlungen (sangkepan desa) finden in der Regel einmal im balinesischen Monat zu 35 Tagen - falls möglich im pura bale agung bzw. im pura desa - statt. Nebst Routinegeschäften wie Verwaltung und Unterhalt von profanem und sakralem Dorfeigentum, besteht eine der Hauptpflichten in der Verteilung der Verantwortlichkeiten und in der Organisation für die einmal im 210Tage-Jahr stattfindenden "Geburtstagsfeier" (piodalan) der Dorftempel, mit welchen die Purifizierung des Dorfterritoriums und der ganzen Tempelkongregation angestrebt wird. Auch in diesem Fall regelt das Dorf die religiös-rituellen Aspekte und delegiert die dazu notwendigen vorbereitenden Arbeiten an seine Banjargemeinschaften. In Dörfern, in denen die Anzahl der krama desa so groß geworden ist, daß sich Vollversammlungen kaum mehr realisieren lassen, werden Vertreter der Dorfgemeinschaften zu den Sitzungen abgeordnet, so beispielsweise die Banjarvorsteher (klian banjar), die Assistenten des bandesa und Tempelpriester (pamangku).

Dörfer als politisch-administrative Einheiten (ind.: desa dinas; bal.: perbekalan)setzen sich nicht nur aus verschiedenen banjar; sondern oft sogar aus mehreren desa adat zusammen. Dabei stimmen die Grenzen der politischen Gemeinden nicht immer mit denjenigen von Adatdörfern überein. Dem kepala desa (ind.) oder perbekel (bal.) fällt die Aufgabe zu, die Implementierung von Verfügungen und Erlassen der nationalen und regionalen Regierung in seinem Befugnisbereich zu überwachen.

\subsubsection{Bewässerungsgemeinschaft}

$\mathrm{Zu}$ den wichtigsten Institutionen agrarökonomisch/religiösen Charakters in Naßreisgebieten zählen die seit über tausend Jahren bestehenden Subakgemeinschaften (sekaha subak). Deren Mitglieder umfassen sowohl alle Landbesitzer als auch alle Pfandnehmer, Pächter und Teilbauern von Parzellen, die innerhalb ein und desselben subak liegen. Ein subak kann als Schwerkraftbewässerungskomplex definiert werden, der sein Wasser von einer bergwärts gelegenen, gemeinsamen Wasserspeisung (z. B. von einem durch einen Damm gestauten Fluß) bezieht, dieses über ein ausgeklügeltes Verteilungssystem in die zahlreichen, von Erdwällchen begrenzten, leicht geneigten Parzellen leitet und von Terrasse zu Terrasse stufenweise nach unten abfließen läßt. Abhängig von topographischen und hydrographischen Bedingungen schwanken die Flächen der gegen 1300 balinesischen subak stark. An steilen Flußböschungen können sie eine Hektare unterschreiten, in den Gunstzonen der großen Alluvialebenen hingegen mehrere Quadratkilometer große Bewässerungseinheiten umfassen. Da die einzelnen, von einem Fluß gespeisten subak oft wiederum untereinander verbunden sind, gilt es, nicht nur Regelungen über Wasseranrechte innerhalb eines Bewässerungskomplexes, sondern auch zwischen solchen zu schaffen und einzuhalten. Konflikte zwischen verschiedenen Subakgemeinschaften können dann die Folge sein, wenn in niederschlagsarmen Jahren flußaufwärts gelegene Bewässerungseinheiten so viel Wasser abzapfen, daß die Wasserzufuhr für tiefer liegende beeinträchtigt wird. In vorkolonialer Zeit wurde zur Schlichtung solcher Streitfälle der König oder dessen Statthalter als nicht direkt in Subakbelange involvierter und somit neutraler Schiedsrichter angerufen (H. GEERTZ und C. GEERTZ 1975: 20). Herrscher fungierten aber nicht nur als Schlichter für Bewässerungskonflikte, sondern veranlaßten ebenso den Bau und die Überwachung königlicher Dämme im Ober- und Mittellauf von Flüssen und stimulierten die dafür notwendigen Rituale. Da Naßreis die entscheidende Nahrungsressource wie auch Quell königlicher Revenuen war, hing die Wohlfahrt der Bevölkerung und der Reichtum der Herrscher in entscheidendem Maß von der Kontrolle der Wasserzufuhr ab. Machtrivalitäten zwischen Herrschern - so etwa zwischen Mengwi und Badung - drückten sich denn auch oft in Kämpfen um Wasserfassungen aus.

Da subak oft gemeindeübergreifende Areale abdecken, setzt sich deren Mitgliedschaft aus Angehörigen verschiedener Dorfgemeinschaften zusammen. Mit Ausnahme der Fälle, in denen Dorfrituale vom subak tangiert werden, sind Dorf und subak getrennte adatrechtliche Institutionen. Besitzt und/oder bewirtschaftet ein Bauer Parzellen in zwei oder mehreren subak, ist er obligatorisch Mitglied der jeweiligen Bewässerungsgemeinschaften. Unbesehen von Kastenzugehörigkeit und anderen Statuspositionen, aber auch unabhängig von der Größe ihrer Besitz- und/oder Nutzflächen im subak und ihres Wasseranspruchs, sind alle bei Entscheidungsfindungen anläßlich verbindlicher Sitzungen gleichberechtigt. Die Höhe der auferlegten Wasserschaftssteuern wie auch die zu erbringenden Dienstleistungen richten sich hingegen nach dem Wasserbezug bzw. nach der Fläche der Bewässerungsparzellen. Vorschriften für die Mitgliedschaft, Pflichten und Rechte der Mitglieder einer Subakgemeinschaft sind in Satzungen festgelegt, die aus Prestigegründen oft im Namen des Königs aufgesetzt oder durch diesen bestätigt worden sind (SCHAAREMAN 1986: 121). Sie enthalten nicht nur Klauseln für organisatorische und technische Bereiche, sondern regeln auch subakbezogene Sakralbelange. Zu ersteren zählen Vorschriften über die Wasserverteilung, Steuern und andere Abgaben, zu erbringende Arbeitsleistungen, Sanktionen bei Wasserdiebstählen oder bei Beschädigungen bzw. Vernachlässigungen von Bewässerungsanlagen und Bestimmungen zur Schlichtung von Streitigkeiten unter den Mitgliedern (BUNDSCHU 1987: 41). Der sakrale Aspekt einer Subakgemeinschaft kommt nicht allein in der regelmäßigen Darbietung von Opfergaben und der gemeinsamen Durchführung von religiösen Feiern in Bewässerungstempeln zum Ausdruck, sondern auch darin, daß ähnlich wie im Falle des desa adat - eine Verletzung der Subaksatzungen als Verstoßgegen die göttliche Ordnung 
gewertet wird und die kosmische Harmonie zu destabilisieren droht: Bei Verfehlungen eines Individuums werden negative Konsequenzen, wie beispielsweise Mißernten, für die ganze Gemeinschaft befürchtet.

Ausschlaggebendes Organ der Subakgemeinschaft ist die Mitgliederversammlung. Im Verhinderungsfall ist das Mitglied angehalten, einen Stellvertreter zu bestimmen. Sitzungen finden entweder routinemäßig einmal im 35-Tage-Monat statt oder werden nach Bedarf bei anfallenden Geschäften anberaumt. Ort der Zusammenkunft ist der Subaktempel. Dem von den Mitgliedern gewählten oder im Rotationsverfahren bestimmten Vorsteher (klian subak) stehen verschiedene Assistenten zur Seite. Zu den Aufgabenbereichen des Subakvorstehers zählen die Einberufung der Mitgliederversammlung und deren Leitung. Er führt die Mitgliederliste und registriert GröBe und Lage der Parzellen sowie deren Besitzer bzw. Bewirtschafter. Zu seinem Pflichtenheft gehört ferner die Überwachung von Arbeiten am subak und die Organisation von Patrouillengängen zur Überprüfung der Einhaltung zugesprochener Wasserlimiten. Da staatlich initiierte landwirtschaftliche Modernisierungsprogramme vor allem auch den Naßreisanbau tangieren, zeichnet ein klian subak ebenso für die Implementierung von Regierungserlassen durch die Subakgemeinschaft verantwortlich. Bei Verstößen gegen die Subaksatzungen legt der klian subak und/oder die Versammlung das Strafmaß fest. In der Regel handelt es sich um Geldbußen, im Extremfall um den Entzug des Wasserrechts (BUNDSCHU 1987: 43 f.). Die Versammlung selbst befindet über Planung und Realisierung von Arbeiten an Bewässerungsinstallationen, die Ausbesserung von Terrassen, das Timing der Naßreiskultivierung und organisiert die Vorbereitung religiöser Gemeinschaftsrituale. Sie bespricht auch Anpassungen der Subaksatzungen an veränderte externe Rahmenbedingungen.

Der sekaha subak bezieht seine Einkünfte aus Eintrittsbeiträgen von Neumitgliedern, flächen- bzw. wasseranteilsbezogenen Jahresbeiträgen sowie von außerordentlichen Abgaben. Weitere Einkünfte fließen aus Bußengeldern und Freikaufbeiträgen von Mitgliedern zu, die ihren Anteil am Gemeinschaftswerk nicht durch eigene Arbeit, sondern monetär abgelten. Anfallende Arbeiten im engeren Bereich der Bewässerung werden von einer speziellen Wasserschaftsgemeinschaft (sekaha yeh) wahrgenommen, der ein pekaseh vorsteht. Subakmitglieder, die dieser Wasserschaftsgemeinschaft nicht angehören, kompensieren durch den sekaha yeh erbrachte Arbeiten mit einer Wassersteuer (sawinih) (SCHAAREMAN 1986: 121). Die Einnahmen der Subakgemeinschaft fließen dem Subakfonds zu. Zum Vermögen zählen die materielle Infrastruktur des Bewässerungskomplexes (sofern sie nicht Staatsbesitz ist) sowie technische Gerätschaften. Falls einem Subaktempel Felder (laba pura) zugesprochen sind, werden Teile der Erträge anläßlich spezifischer Tempelfeiern redistribuiert. Dem gleichen Zweck dienen Abführungen eines gewissen Ernteanteils an die Bewässerungsgemeinschaft. Auch in agraren Belangen kommt somit die Einbindung des Balinesen in ein Kollektiv zum Ausdruck, dessen Bestreben es ist, durch adatkonformes Handeln innerhalb definierter Grenzen Profanes und Sakrales in Einklang zu bringen und somit zu harmonisieren.

\section{Anmerkungen}

' Auch die Gesellschaftsorganisation der sasak boda-Dörfer auf der östlichen Nachbarinsel Lombok weist verwandte Merkmale mit den bali aga auf, ohne daß zwischen den beiden direkte Beziehungen bestanden hätten. Innerhalb von Lombok selber liegen die heutigen Agasiedlungen bis über $80 \mathrm{~km}$ isoliert voneinander in ganz unterschiedlichen Naturlandschaften. Nur die wenigsten auf der Nordabdachung der zentralen Gebirgskette lebenden boda (sasak aga) wissen um die Existenz anderer Bodadörfer an der auf dem Landweg schwer zugänglichen südwestlichen Küste (LEEMANN 1989: 9-20).

${ }^{2}$ Vgl. dazu den Prozeß der Entwicklung von Nutzrechten der Bauern auf Land in Kommunalbesitz bis hin zum Privateigentum an Boden (SUDIYAT 1981: 1-5).

${ }^{3}$ Es kann der Fall sein, daß sich Bewohner von Bergdörfern gelegentlich als Angehörige mehrerer Abstammungsgruppen ausgeben.

${ }^{4} \mathrm{Vgl}$. analog dazu die volksetymologische Mißinterpretation des Wortes boda durch "fortschrittliche" sasak, die "boda" vom indonesischen Wort bodoh (dumm) oder gar vom Sasakwort buduh (verrückt) herleiten. Ähnlich diskriminierend ist die Gleichsetzung von sasak boda mit "Waldmensch" oder "Bergmensch".

${ }^{5}$ bedaulu (syn.: beda muka) = "verschiedenes Gesicht". Nachkommen des Dalem Bedaulu tragen bis heute den Namen Muka.

${ }^{6}$ Schriftliche Hinweise zur Majapahit-Zivilisation finden sich in dem in Versform geschriebenen Werk Nagarakretagama, verfaßt vom javanischen Poeten und Historiographen MPU PRAPANCA in der zweiten Hälfte des 15. Jahrhunderts. Eine weitere Quelle ist das etwas später, wahrscheinlich zwischen 1478 und 1486 in Prosa geschriebene Pararaton (SLAMETMULYANA 1979).

${ }^{7}$ wauh rauh (bal.) = "Soeben angekommen". Auf Lombok ist Dang Hyang Nirartha unter dem Namen Dang Hyang Sangupati, auf Sumbawa unter dem Namen Pangeran Semeru bekannt.

${ }^{8}$ Je nach Quelle variieren Zahl und Namen der Töchter und Söhne von Dang Hyang Nirartha. Unterschiede in den Namen können sich beispielsweise daraus ergeben, daß bei Priesterweihen (resi yadnya) Personen einen neuen Namen annehmen.

${ }^{9}$ disengguh padanda (bal.) $=$ "mit dem Priester verwechselt". Somit drückt der Name der Titelgruppe sengguhu die Verwechslung aus.

${ }^{10}$ Die Version zur Titelgruppenbildung der sengguhu, wie sie vom balinesischen "Forschungsteam zur Geschichte von Ida Dang Hyang Nirartha" vorgelegt wird, unterscheidet sich von obiger Fassung in Zeit und Ort, nicht aber in der Kernidee der Verwechslung eines sudra mit einem javanischen Brahmanenpriester als Heilsbringer und der Zuerkennung der Leitung von Zeremonien zur Beschwichtigung chthonischer Mächte (upacara percaruan) an die genannte Titelgruppe. Gemäß Deutung des Forschungsteams soll der javanische Priester Dang Hyang Nirartha auf seiner Missionierungsreise durch Bali, Lombok und Sumbawa (15./16. Jahrhundert) im 
Dorf Kapal haltgemacht und von dessen bandesa vernommen haben, daß I Guto - ohne Priester zu sein - puja waliZeremonien im pura Sada vorzustehen pflege. Daraufhin hätte inm Dang Hyang Nirartha die Leitung gottbezogener Rituale (dewa yadnya) strikte untersagt, I Guto und seinen Nachkommen aber erlaubt, als sengguhu Zeremonien zur Beschwichtigung von Dämonen führen zu dürfen.

"Der Vorsteher des Kantor Agama Hindu in Denpasar begründet das Hühnerfleischtabu für padanda damit, daß es zu vermeiden gelte, daß der schlechte Charakter gackernder und streitsüchtiger Hühner auf den Priester übergehe. Positiver ist die balinesische Wertung von Enten, die als friedliebende und kluge Tiere eingestuft werden.

${ }^{12}$ bukpadan (bal.): "Staub des Fußes". Die gleiche Bedeutung wohnt der aus der Kawisprache stammenden Ansprechformel für hohe Persönlichkeiten: paduka inne, wörtlich übersetzt "Schuh". Cf. zur Regierungszeit Soekarno: "Paduka Yang Mulia Presiden" oder während der Kolonialzeit für den niederländischen Residenten: "Paduka Kanjeng Tuan Besar Residen".

${ }^{13}$ Ein adatbewußter Balinese hat "drei Lehrern der Weisheit" (triguru wisesa) die Ehre zu erweisen: seinen Eltern, Lehrern und dem Herrscher (bzw. der Regierung).

${ }^{14}$ Beim Ableben eines commoners fällt das ganze Dorf in den Zustand der Unreinheit (sebel), im Falle des Hinschiedes eines Adeligen sogar eine ganze Region. Im ostbalinesischen Dorf Jangu etwa dauert diese Zeitspanne der Unreinheit 25 Tage, während der eine ganze Reihe religiös-ritueller Veranstaltungen tabu sind.

${ }^{15}$ nyepi = "ruhig sein". Am nyepi-Tag darf kein Inselbewohner seinen Familienhof (und kein Tourist sein Hotel) verlassen. Nyepi, der Tag physischer Inaktivität, ist der Tag der Einkehr und Besinnung und leitet das neue Jahr ein. Da nach landläufiger Auffassung die Leidenschaften und Krankheiten die Menschen in Form von Hitze heimsuchen, ist es am balinesischen Neujahrstag bei Strafe verboten, Feuer zu entfachen.

${ }^{16}$ Gelegentlich - wie etwa im Falle des Dorfes Sempidi - sind der pura puseh und der pura desa in einem Tempel zusammengefaßt.

\section{Literatur}

ALISJAHBANA, S. T. (1966): Indonesia: Social and Cultural Revolution, Kuala Lumpur, Singapore, London, Melbourne.

BUNDSCHU, I. (1987): Kooperation und landwirtschaftliche Entwicklung auf Bali/Indonesien, Hamburg.
GEERTZ, C. (1959): Form and variation in Balinese village structure. In: American Anthropologist, 61, 991-1012.

GEERTZ,H. and GEERTZ, C. (1975): Kinship in Bali, Chicago, London.

GUERMONPREZ, J.-F. (1980): L'organisation villageoise à Bali. In: ASEMI, XI, 1-4, 37-54.

GUERMONPREZ, J.-F. (1984): Les Pandé de Bali. La Formation d'une Caste et l'Imaginaire d'un Titre, Paris.

HANNA, W. A. (1976): Bali Profile. People, Events, Circumstances (1001-1976), New York.

HOBART, M. (1980): Ideas of Identity. The Interpretation of Kinship in Bali, Denpasar.

HOWE, L. E. A. (1989): Hierarchy and Equality: Variations in Balinese Social Organization. In: Bijdragen tot de Taal-, Landen Volkenkunde, 145/1, 47-71.

KORN, V. E. (1932): Het Adatrecht von Bali, 's-Gravenhage.

LEEMANN, A. (1976): Bali - Auswirkungen des balinesischen Weltbildes auf verschiedene Aspekte der Kulturlandschaft und auf die Wertung des Jahresablaufs. In: Ethnologische Zeitschrift Zürich, II, 27-65

LEEMANN, A. (1979): Bali, Innsbruck, Frankfurt a. M.

LEEMANN, A. (1989): Internal and External Factors of Sociocultural and Socio-economic Dynamics in Lombok (Nusa Tenggara Barat), Zürich.

RÖLL, W. und LEEMANN, A. (1987): Agrarprobleme auf Lombok. Untersuchungen zur Wirtschafts- und Sozialstruktur in Nusa Tenggara Barat, Indonesien, Hamburg

SCHAAREMAN, D. (1986): Tatulingga: Tradition and Continuity. An Investigation in Ritual and Social Organization in Bali, Basel.

SCHULTE NORDHOLT.H. (1986): Bali: Colonial Conceptions and Political Change 1700-1940. From Shifting Hierarchies to "fixed Order". In: casp, 15.

SLAMETMULYANA (1979): Nagarakretagama dan tafsir sejarahnya, Jakarta.

SUDIYAT, I. (1981): Hukum Adat, Sketsa Asas, Yogyakarta.

SUGRIWA, I G. Bgs. (1990): Babad Pasek, Denpasar

SWELLENGREBEL, J.L. ed. (reprint 1984): Bali: Studies in Life, Thought, and Ritual, Dordrecht, Cinnaminson. 